Ann. Sc. forest., 1980, 37 (2), 109-133.

\title{
Variabilité intraspécifique de l'épicéa : stabilité et homogénéité des provenances et régions de provenances allemandes
}

\author{
Catherine CHRISTOPHE \\ Station d'Amélioration des Arbres forestiers, \\ Centre de Recherches forestières d'Orléans, I.N.R.A., \\ Ardon, 45160 Olivet
}

\begin{abstract}
Résumé
L'exploration de la variabilité intraspécifique de l'épicéa (Picea abies) dans son aire naturelle à partir de deux dispositifs installés en France est confrontée avec des résultats établis en Allemagne sur des dispositifs équivalents.

Au niveau de grandes régions géographiques, la supériorité des Beskides (Pologne, Tchécoslovaquie), de la Roumanie et du Nord-Est polonais pour des critères de vigueur et de tardiveté du débourrement se retrouve dans les deux pays.

Au niveau des provenances, de fortes interactions provenance $\times$ station sont parfois signalées pour les critères de vigueur.

En vue d'une utilisation commerciale pour les reboisements en France, une attention particulière est portée aux provenances et régions de provenances allemandes ; l'accent est mis sur l'hétérogénéité de quelques provenances d'une part et de la majorité des régions représentées d'autre part. La notion de "région », déjà critiquée par les forestiers allemands, est donc remise en question dans la mesure où la trop grande superficie de certaines et le mélange de peuplements naturels et artificiels dans d'autres ne permettent pas d'assurer l'homogénéité d'un matériel issu de ces régions.
\end{abstract}

\section{Introduction}

Toute stratégie d'amélioration d'une espèce forestière commence par l'exploration de la variabilité naturelle de cette espèce. Dans le cas de l'épicéa (Picea abies) cette variabilité intraspécifique est déjà relativement bien connue des sélectionneurs forestiers.

Cette espèce étant largement utilisée en reboisement hors de son aire naturelle, il paraît nécessaire de connaître également la stabilité du comportement des provenances installées dans divers pays.

L'intérêt d'étudier une plantation comparative de provenances d'épicéa en France est actuellement double :

- d'une part comparer globalement le comportement de ces provenances avec celui observé en Allemagne, 
- d'autre part étudier l'homogénéité des régions de provenances allemandes dont les limites actuelles sont remises en cause par les améliorateurs allemands euxmêmes.

Ces sources de graines peuvent en effet jover un rôle non négligeable dans l'approvisionnement des reboisements français compte tenu des liens commerciaux entre pays d'une part et des obligations découlant de la réglementation C.E.E. d'autre part.

\section{1. - Description de l'expérience}

\section{1. - Matériel}

La présente étude porte sur cent provenances récoltées pour la plupart dans l'aire naturelle de l'épicéa regroupant quarante provenances de la zône alpine (Autriche, Suisse, France), vingt-huit provenances allemandes, six provenances nordiques (Suède, Finlande), quinze provenances de la région des Beskides (Moravie, Sudètes), sept provenances du Nord-Est et Centre Polonais, une provenance roumaine, deux provenances russes et enfin une provenance non identifiée.

La description ef la localisation de ces provenances sont présentées en annexe.

Les semis 2-0, élevés dans la pépinière de l'Institut d'Amélioration des arbres forestiers de Hann Münden dans le Hesse (Allemagne Fédérale) sont arrivés en France en très mauvais état, suite à des conditions de transport très difficiles. Repiqués en pépinière d'Amance au printemps 1964, ils n'ont pu être installés sur le terrain qu'à l'automne 1966, après avoir subi une crise au repiquage considérable : les plants avaient donc déjà 5 ans (2-3) lorsqu'ils furent plantés en forêt domaniale d'Amance (altitude $240 \mathrm{~m}$, pluviométrie annuelle $700 \mathrm{~mm}$ ), selon un dispositif en blocs complets à parcelles monoarbres (30 blocs).

Les cent provenances représentées ici ne sont qu'un échantillon de l'ensemble des cinq cent trente provenances internationales étudiées en Allemagne même dans quatre stations différentes (Weisgerber ef al., 1976).

\section{2. - Caractères mesurés}

- Avant la plantation (1966), des mesures de diamètre au collet et de hauteur tolale ont été effectuées sur tous les plants.

- Compte tenu du mauvais état initial des plants rendant encore plus probable la crise de transplantation fréquente chez l'épicéa, aucune mesure n'a été effectuée sur le terrain jusqu'en 1970. A partir de 1971, soit 10 ans depuis la graine (5 ans après la plantation) et jusqu'en 1978, soit à 17 ans, tous les plants ont été mesurés pour leur hauteur totale : on dispose donc également des mesures de pousses annuelles ef de pousses cumulées sur plusieurs années.

- En 1978, une mesure de la circonférence à $1,30 \mathrm{~m}$ a été réalisée sur fous les plants.

- A côté de ces critères de vigueur, une notation du débourrement végétatif 
a été établie en trois passages les $17 \mathrm{mai}, 25$ mai et 4 juin 1973. A chaque passage une note de 0 à 5 était attribuée au bourgeon terminal de chaque plant, selon un code 0-5:

0 : bourgeon dormant

1 : bourgeon en rosette entière (écailles non déchirées)

2 : apparition des aiguilles (déchirement ou disparition totale des écailles, pas d'allongement des aiguilles)

3 : allongement des aiguilles, pousse encore gonflée

4 : stade pinceau : allongement aiguilles serrées

5 : allongement maximum, aiguilles écartées.

A partir de cette notation individuelle, deux pourcentages ont été également pris en comple : pourcentage de plants ayant une note supérieure à 1 lors du premier passage, pourcentage de plants ayant une note au moins égale à 4 lors du dernier passage.

\section{3. - Les conditions climatologiques}

Il paraît intéressant de relever les pluviométries durant la période étudiée, dans la mesure où elle inclut deux années (1975 et 1976) remarquables par leur sécheresse : les données nous sont fournies par la station de Bioclimatologie forestière, poste d'Amance (« Hiver» inclut les mois d'octobre à avril).

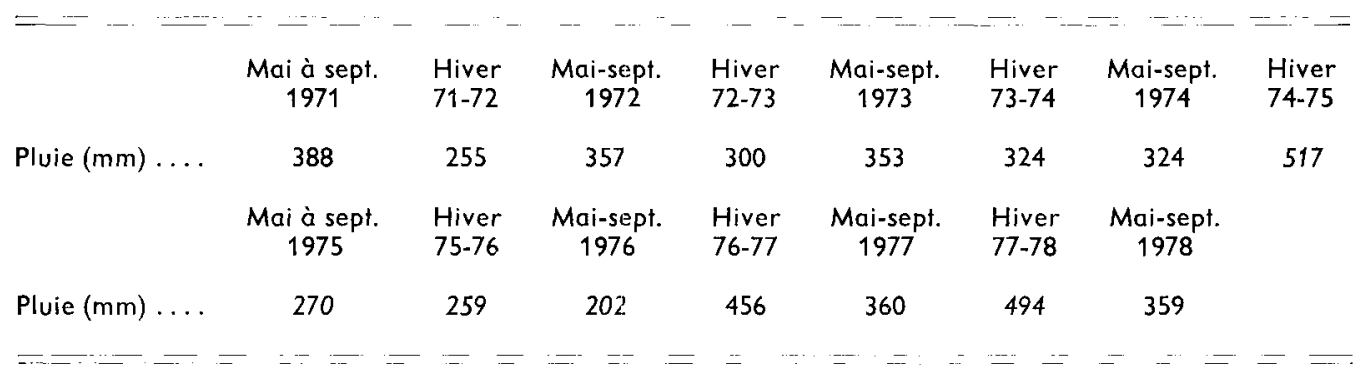

II faut relever, à côté de la régularité des années 1971 à 1974, les deux hivers 1974-75 et 1977-78 particulièrement humides, tandis que la période mai 1975 à septembre 1976 se singularise par une exceptionnelle sécheresse : ceci se répercute au niveau des pousses annuelles puisque 1975 et 1978 traduisent une forte croissance $(69$ et $88 \mathrm{~cm})$ alors que les pousses des années 1976 et $1977(51$ et $53 \mathrm{~cm})$ répercutent la sécheresse des deux années précédentes.

\section{4. - Traitement des données}

Au niveau de la comparaison des provenances, un modèle linéaire classique à effets fixés, prenant en compte le facteur bloc et le facteur provenance, a été appliqué à chacun des caractères afin d'étudier la signification des facteurs.

Le tableau 1 résume l'analyse de variance utilisée dans chaque cas. 


\section{TABLEAU 1}

Analyse de variance non orthogonale ò 2 facteurs

The two-way crossed analysis of variance with unbalanced data

\begin{tabular}{|c|c|c|c|c|}
\hline $\begin{array}{l}\text { Source de variation } \\
\text { (origin of variation) }\end{array}$ & $\begin{array}{l}\text { DDL } \\
(d . f)\end{array}$ & $\begin{array}{l}\text { Somme } \\
\text { des carrés } \\
\text { (sum } \\
\text { of squares) }\end{array}$ & $\begin{array}{l}\text { Carrés } \\
\text { moyens } \\
\text { (mean } \\
\text { square) }\end{array}$ & $\begin{array}{l}\text { Tests } F \\
(F)\end{array}$ \\
\hline $\begin{array}{l}\text { Facteur bloc (non ajusté) (block) } \ldots \ldots \ldots \ldots \ldots \\
\text { Facteur provenance (ajusté) (provenance (after } \\
\text { block)) } \ldots \ldots \ldots \ldots \ldots \ldots \ldots \ldots \ldots \ldots \ldots \ldots \ldots \ldots \ldots \ldots \ldots \ldots\end{array}$ & 99 & $\begin{array}{l}R(b / \mu) \\
R(p / \mu, b)\end{array}$ & CMP & $F_{D}=\frac{C M P}{C M E}$ \\
\hline Résiduelle (residual error) $\ldots \ldots \ldots \ldots \ldots \ldots$ & 2661 & SSE & CME & \\
\hline Facteur bloc (ajusté) (block (after provenance)) .. & 29 & $R(b / \mu, p)$ & $\mathrm{CMB}$ & $F_{\mathrm{b}}=\frac{\mathrm{CMB}}{\overline{C M E}}$ \\
\hline $\begin{array}{l}\text { Facteur provenance (non ajusté) (provenance) } \ldots \\
\text { Résiduelle (residual error) } \ldots \ldots \ldots \ldots \ldots \ldots \ldots\end{array}$ & $\begin{array}{r}99 \\
2661\end{array}$ & $\begin{array}{l}R(p / \mu) \\
\text { SSE }\end{array}$ & CME & \\
\hline Total (total) & 2789 & $\begin{aligned} \mathbf{S S T} & =\mathbf{S S} \\
& =\mathbf{S S}\end{aligned}$ & $\begin{array}{l}+R(b / \mu) \\
+R(P / \mu)\end{array}$ & $\begin{array}{l}R(p / \mu, b) \\
R(b / \mu, p)\end{array}$ \\
\hline
\end{tabular}

Termes R( ) introduits par Searle (1971) : $R(b / u)$ est la réduction des sommes de carrés due à la prise en compte de l'effet bloc ignorant l'effet provenance; $R(p / \mu, b)$ est la réduction due d̀ la prise en compte de l'effet provenance ajusté aux effets blocs.

The $R($ ) come from Searle (1971); $R(b / \mu, b)$ is the sum of squares due to fitting block after the general mean $\mu ; R(p / \mu, b)$ is the sum of squares due to fitting provenances after bloc and mean.

L'analyse précédente permettant de tester la signification de l'effet provenance pour un caractère donné, nous avons eu ensuite recours à l'analyse multivariable classique pour permettre une représentation graphique simplifiée des provenances: une première analyse portera uniquement sur tous les critères de vigueur (hauteurs totales, pousses, circonférence), une seconde sur les critères de débourrement et une troisième enfin reprendra l'ensemble de tous les critères (vigueur et débourrement).

Tous les traitements utilisant des programmes de la Station de Biométrie de Nancy ont été effectués sur l'ordinateur (IRIS 80 ) du Centre Interuniversitaire de Calcul d'Orléans-La Source.

\section{2. - Résultats généraux}

\section{1. - Etude des critères de vigueur}

Pour tous les caractères de hauteurs totales, pousses annuelles et pousses cumulées, circonférence, les effets blocs comme les effets provenances sont hautement significatifs. Les valeurs des tests $F$ calculées d'après le tableau 1 sont reportées dans le tableau 2.

Les fortes valeurs des $\mathrm{F}$ et leur évolution méritent que l'on regarde de près l'action des années sur l'effet bloc avant de s'intéresser au classement des provenances.

\subsection{Etude du milieu.}

Les valeurs du tableau 2 indiquent que l'effet bloc sur les hauteurs totales, bien que restant très significatif, a tendance d̀ diminuer régulièrement de 1971 à 1978 . On 
TABLEAU 2

Valeurs de $F_{\mathrm{p}}$ ef $\mathrm{F}_{\mathrm{b}}$ en fonction du temps

Values of $F_{p}$ and $F_{b}$ with years

\begin{tabular}{rrrrrrrrrrrrrrrrr} 
& $H 72$ & $H 73$ & $H 74$ & $H 75$ & $H 76$ & $H 77$ & $H 78$ & P72 & P73 & P74 & P75 & P76 & P77 & P78 \\
\hline$F_{\mathrm{b}}$. & 41 & 39 & 34 & 28 & 31 & 30 & 23 & 34 & 14 & 10 & 9 & 21 & 20 & 11 \\
$F_{p}$. & 8 & 8,4 & 8,6 & 8,8 & 9 & 9,3 & 9,9 & 5,6 & 4,6 & 5 & 4,5 & 3 & 3 & 3 \\
\hline
\end{tabular}

«H72 »= Houteur totale 1972 (11 ans depuis la graine, 6 ans après la plantation).

«P72 »= Pousse 1972 (11e année depuis la graine).

« $\mathrm{H} 72$ » = Total height 1972 (11 years from seed, 6 years after plantation).

«72 » == Height increment 1972 (11th year from seed).

retrouve également cette tendance au niveau des pousses annuelles jusqu'en 1975, perturbée en 1976 et 1977 par la sécheresse des années 1975 el 1976. L'effet bloc diminue à nouveau sur la pousse de l'année 1978.

Si l'on compare les classements des blocs obtenus sur les pousses annuelles successives, on constate de très fortes interactions blocs-année. Cependant les changements de classement observés semblent s'opérer progressivement et concernent des groupes de blocs voisins sur le terrain : ainsi les blocs 1 à 7 , très vigoureux au départ, restent les plus vigoureux au niveau des hauteurs totales 1978 : situés dans la partie haute du dispositif, ils profitent en plus d'un effet lisière protecteur. Par contre, très vigoureux au niveau des pousses 1972 et 1973, ils atteignent la moyenne générale en 1975, et après une faible remontée en 1976, tombent nettement en-dessous de la moyenne en 1977 et 1978, l'effet lisière pouvant désormais leur être néfaste. Le comportement des blocs 20 à 25 (dans la partie basse du dispositif) esf tolalement inverse : peu vigoureux jusqu'en 1974, ils semblent se sortir progressivement d'une concurrence arbustive intense, et très vigoureux depuis 1975, ils viennent en tête du classement en 1978.

Cette variation du bloc avec le temps est importante à signaler : elle remet en cause la notion de fertilité du bloc, considérée habituellement comme définitive. La fertilité du bloc est au contraire évolutive : ainsi le bloc 15, situé également dans la partie basse et fortement concurrencé par la végétation adventice, qui n'atteignait qu'en 1978 la hauteur moyenne $(4,6 \mathrm{~m})$ qu'avait obtenue déjà en 1975 le bloc 6, révèle en 1978 une forte pousse annuelle $(95 \mathrm{~cm})$ alors que le bloc 6 pousse peu $(79 \mathrm{~cm})$.

Cette évolution se traduit aussi au niveau des corrélations entre moyennes de blocs sur les caractères de vigueur : d'une année à l'autre, les corrélations entre hauteurs totales restent très élevées mais diminuent régulièrement (entre 1971 et 1972, la corrélation entre blocs esł de 0,99, entre 1971 et les différentes années successives elle décroît doucement pour afteindre 0,87 entre 1971 et 1978).

Au niveau des pousses annuelles, les corrélations sont très variables, dues aux changements de classement de fertilité des blocs (voir tabl. 3). 
TABLEAU 3

Corrélations entre pousses annuelles ou niveau moyennes de blocs Correlations between annual height increment (at bloc means level)

\begin{tabular}{|c|c|c|c|c|c|c|}
\hline & P72 & P73 & P74 & P75 & P76 & P77 \\
\hline $\begin{array}{l}\text { P73 } \\
\text { P74 }\end{array}$ & $\begin{array}{l}0,88 \\
0,74\end{array}$ & 0,87 & & & & \\
\hline P75 & 0,17 & 0,40 & 0,72 & & & \\
\hline P76 & 0,61 & 0,61 & 0,79 & 0,76 & & \\
\hline P77 & $-0,08$ & 0,19 & 0,49 & 0,87 & 0,59 & \\
\hline P78 & $-0,78$ & $-0,55$ & $-0,3$ & 0,34 & $-0,12$ & 0,59 \\
\hline
\end{tabular}

« P72 » -- Pousse 1972 (11" année depuis la graine, 6c année depuis la plantation).

«P72»- Height increment 1972 (11th year from seed).

Mise à part la pousse de l'année 1976 qui conjugue les effets de la sécheresse 1975 et de la sécheresse de 1976 elle-même, on retrouve sur le tableau 3 la diminution régulière du coefficient de corrélation du haut en bas du tableau, jusqu'à une corrélation - de 0,78 sur les moyennes de blocs entre les pousses 1972 et 1978 . II sera intéressant de poursuivre cette étude du milieu afin de suivre cette évolution de la fertilité des blocs.

\subsection{Efude des provenances.}

Remarques préliminaires.

Le classement des provenances en 1978 est très proche de celui qui avait été établi à la sortie de la pépinière en 1966 (la corrélation entre les 2 années est de $0,87)$. Ceci montre qu'après 3 ans de repiquage, les plants étaient sortis de la crise qu'ils avaient subie lors de leur transport entre l'Allemagne et la France. II n'est donc pas aberrant d'inclure les données de pépinière (hauteur ef diamètre au collet) dans l'ensemble des critères de vigueur au niveau provenance tout au moins.

En reprenant les résultats obtenus en Allemagne, on constate qu'à âge égal la vigueur générale des plants semble plus grande à Amance qu'en Allemagne. L'étude allemande concernant un plus grand échantillon que celui étudié ici, les différences de vigueur pourraient s'expliquer par ces différences d'échantillonnage. Cependant Weisgerber et al. (1977) donnent les hauteurs totales de six provenances allemandes dans les quatre stations expérimentales allemandes et nous pouvons les comparer avec leurs performances obtenues la même année (1974) à Amance :

\begin{tabular}{|c|c|c|c|c|c|}
\hline Stations & Bayern & Baden-Würtenberg & Niedersachsen & Hesse & Amance \\
\hline $\begin{array}{c}\text { Hauteurs moyennes sur } 6 \\
\text { provenances } \ldots \ldots \ldots \ldots \ldots\end{array}$ & $1,12 \mathrm{~m}$ & $1,57 \mathrm{~m}$ & $1,96 \mathrm{~m}$ & $2,77 \mathrm{~m}$ & $3,07 \mathrm{~m}$ \\
\hline Moyennes générales & $1,03 \mathrm{~m}$ & $1,60 \mathrm{~m}$ & $1,90 \mathrm{~m}$ & $2,88 \mathrm{~m}$ & $3,18 \mathrm{~m}$ \\
\hline
\end{tabular}


Les six provenances expriment donc bien les différences de fertilité entre les quatre stations et confirment la plus forte vigueur observée actuellement à Amance. De plus, il faut noter que la croissance a été beaucoup plus forte depuis la plantation à Amance puisqu'à la sortie de pépinière (1966) les performances allemandes étaient alors plus élevées : les hauteurs moyennes allaient de $37 \mathrm{~cm}$ à $45,6 \mathrm{~cm}$ suivant les pépinières allemandes, elles n'étaient que de $36 \mathrm{~cm}$ en pépinière d'Amance.

\section{Comportement des provenances.}

La stabilité dans le classement pour les critères de vigueur des cent provenances représentées ici est remarquable : les corrélations entre hauteurs totales sont très élevées, on a vu plus haut que dès la pépinière le classement sur les hauteurs totales était fixé. De plus, la hauteur en pépinière ( 5 ans depuis la graine) est un bon prédicteur de la circonférence à $1,30 \mathrm{~m}$ à 17 ans depuis la graine (la corrélation entre les deux caractères est de 0,87 ). Même au niveau des pousses annuelles, les corrélations restent élevées malgré une légère baisse quand elles impliquent l'année 1977 (conséquence de la sécheresse 1976).

Corrélations au niveau provenances entre les pousses des années
\begin{tabular}{ccccc}
1972 et 71 & 1973 et 72 & 1974 ef 73 & 1975 ef 74 & 1976 et 75 \\
0,89 & 0,89 & 0,89 & 0,84 & 0,75 \\
1977 ef 76 & 1978 et 77 & 1978 et 76 & 1978 et 75 & \\
0,58 & 0,57 & 0,69 & 0,81 & \\
\hline
\end{tabular}

Quelle que soit leur vigueur moyenne, toutes les provenances autrichiennes sauf une, ont réagi à la sécheresse en produisant une faible pousse en 1977. L'exception est la seule provenance autrichienne de basse altitude $(400 \mathrm{~m})$, elle a au contraire bien poussé en 1977. Néanmoins dès 1978, le classement est rétabli puisqu'avec 1975 la corrélation entre provenances est remontée d̀ 0,81 .

Les valeurs de $F_{p}$ lues dans le tableau 2 indiquent l'importance de l'effet provenance et il semble intéressant d'indiquer un classement des provenances regroupées en grandes régions géographiques, comme l'ont fait Weisgerber ef al., 1977. Nous ne détaillerons cependant pas les provenances allemandes puisqu'elles seront étudiées plus loin.

Le tableau 4 présente donc les valeurs des hauteurs totales ef circonférences à $1,30 \mathrm{~m}$ à 17 ans (la corrélation entre les deux caractères est de 0,95 ) selon les diverses régions. La hauteur moyenne générale est alors de $5,80 \mathrm{~m}$, la circonférence à $1,30 \mathrm{~m}$ est de $21,24 \mathrm{~cm}$. On retrouve classiquement la supériorité des Beskides (Moravie, Pologne), du Nord-Est de la Pologne, de la Roumanie et du Harz. Tout aussi classiquement les régions alpines (France, Suisse, Autriche) et nordiques (URSS, Finlande) se révèlent peu vigoureuses tandis que Allemagne Fédérale et Suède se situent au niveau de la moyenne générale. Au niveau provenance, la variabilité est encore plus grande : la provenance la moins vigoureuse (Suisse) mesure 4,22 $\mathrm{m}$ tandis que la plus vigoureuse (Autriche) atteint $6,82 \mathrm{~m}$, soit une supériorité relative de $62 \mathrm{p} .100$. Ce même gain étant également obtenu sur la circonférence à $1,30 \mathrm{~m}$, il est évident que les retombées sur le volume sont considérables. 
TABLEAU 4

Classement des régions géographiques pour la hauteur 1978 ef la circonférence à $1,30 \mathrm{~m}$ Classification of geographical regions for height 1978 and D.B.H.

\begin{tabular}{|c|c|c|c|c|c|c|c|}
\hline \multirow{2}{*}{$\begin{array}{c}\text { Nombre de } \\
\text { provenances } \\
\text { (number of } \\
\text { provenances) }\end{array}$} & \multirow[b]{2}{*}{$\begin{array}{l}\text { Régions } \\
\text { (regions) }\end{array}$} & \multicolumn{2}{|c|}{ Moyennes (means) } & \multicolumn{2}{|c|}{$\begin{array}{c}\text { Minimum } \\
\text { par région } \\
\text { (minimum value) }\end{array}$} & \multicolumn{2}{|c|}{$\begin{array}{c}\text { Maximum } \\
\text { par région } \\
\text { (maximum value) }\end{array}$} \\
\hline & & $\begin{array}{c}\text { Hauteur } \\
\text { à } 17 \text { ans } \\
\text { (m) } \\
\text { (height } \\
17 \text { years } \\
\text { (m) }\end{array}$ & $\begin{array}{l}\text { Circon- } \\
\text { férence } \\
\text { à } 1,30 \mathrm{~m} \\
(\mathrm{~cm}) \\
(\mathrm{D} . \mathrm{B} . \mathrm{H}) \\
\text { (cm) }\end{array}$ & $\mid \begin{array}{c}\text { Hauteur } \\
\text { à } 17 \text { ans } \\
(\mathrm{m}) \\
\text { (height) }\end{array}$ & $\begin{array}{c}\text { Circon- } \\
\text { férence } \\
\text { à } 1,30 \mathrm{~m} \\
\text { (cm) } \\
\text { (D.B.H.) }\end{array}$ & $\begin{array}{c}\text { Hauteur } \\
\text { à } 17 \text { ans } \\
\text { (m) } \\
\text { (height) }\end{array}$ & $\begin{array}{l}\text { Circon- } \\
\text { férence } \\
\text { à } 1,30 \mathrm{~m} \\
(\mathrm{~cm}) \\
\text { (D.B.H.) }\end{array}$ \\
\hline $\begin{array}{l}3 \\
1\end{array}$ & $\begin{array}{l}\text { Pologne Beskides..... } \\
\text { Roumanie } \ldots \ldots \ldots\end{array}$ & $\begin{array}{l}6,54 \\
6,49\end{array}$ & $\begin{array}{l}24,56 \\
23,88\end{array}$ & 6,41 & 24,15 & 6,66 & 25,2 \\
\hline 9 & Moravie (Tchécoslovaquie) & 6,30 & 22,99 & 5,56 & 18,56 & 6,81 & 25,15 \\
\hline 7 & Pologne N. E. et Centre .... & 6,16 & 22,22 & 5,8 & 19,3 & 6,41 & 23,9 \\
\hline 3 & Harz (Allemagne) ..... & 6,02 & 22,8 & 5,7 & 21,4 & 6,41 & 25 \\
\hline 3 & Tchécoslovaquie ............ & 5,98 & 21,89 & 5,8 & 2,90 & 6,16 & 22,3 \\
\hline 25 & République Fédérale Allemande.. & 5,8 & 20,75 & 4,98 & 18,9 & 6,59 & 24,7 \\
\hline 5 & Suède....................... & 5,8 & 20,75 & 4,98 & 16,2 & 6,39 & 24,6 \\
\hline 23 & Autriche $\ldots \ldots \ldots \ldots \ldots$ & 5,3 & 21,45 & 5,07 & 18,21 & 6,82 & 25,3 \\
\hline 9 & France $\ldots \ldots \ldots \ldots \ldots \ldots \ldots$ & 5,26 & 19,55 & 4,37 & 14,41 & 6,31 & 23,2 \\
\hline 8 & Suisse.................. & 4,97 & 18,36 & 4,22 & 15,49 & 5,46 & 20,11 \\
\hline 2 & U.R.S.S. & 4,63 & 14,22 & & & & \\
\hline 1 & Finlande & 4,46 & 13,99 & & & & \\
\hline
\end{tabular}

Il a paru utile de pouvoir disposer d'une représentation des provenances résumant l'histoire de leur croissance depuis 1966 jusqu'à 1978 : la figure 1 présente le résultał d'une analyse en composantes principales prenant en compte au niveau moyennes de provenances tous les critères de vigueur.

Le premier axe $\left(Y_{1}\right)$, vertical, absorbe 93 p. 100 de la variabilité globale du nuage de points, il est corrélé positivement et fortement avec toutes les variables initiales, elles-mêmes très liées entre elles comme on l'a vu plus haut. Les provenances se rangent donc suivant cet axe selon une composante résumant leur « vigueur globale».

FIG. 1. - Critères de vigueur.

Représentation des provenances suivant les deux premières composantes principales.

Growth traits. Distribution of provenances according to the two first principal components

LÉGENDE : Roumanie
Tchécoslovaquie
Autriche
Pologne
Allemagne
France $\Delta$
Suisse $\nabla$
Suède
Russie-Finlande *.




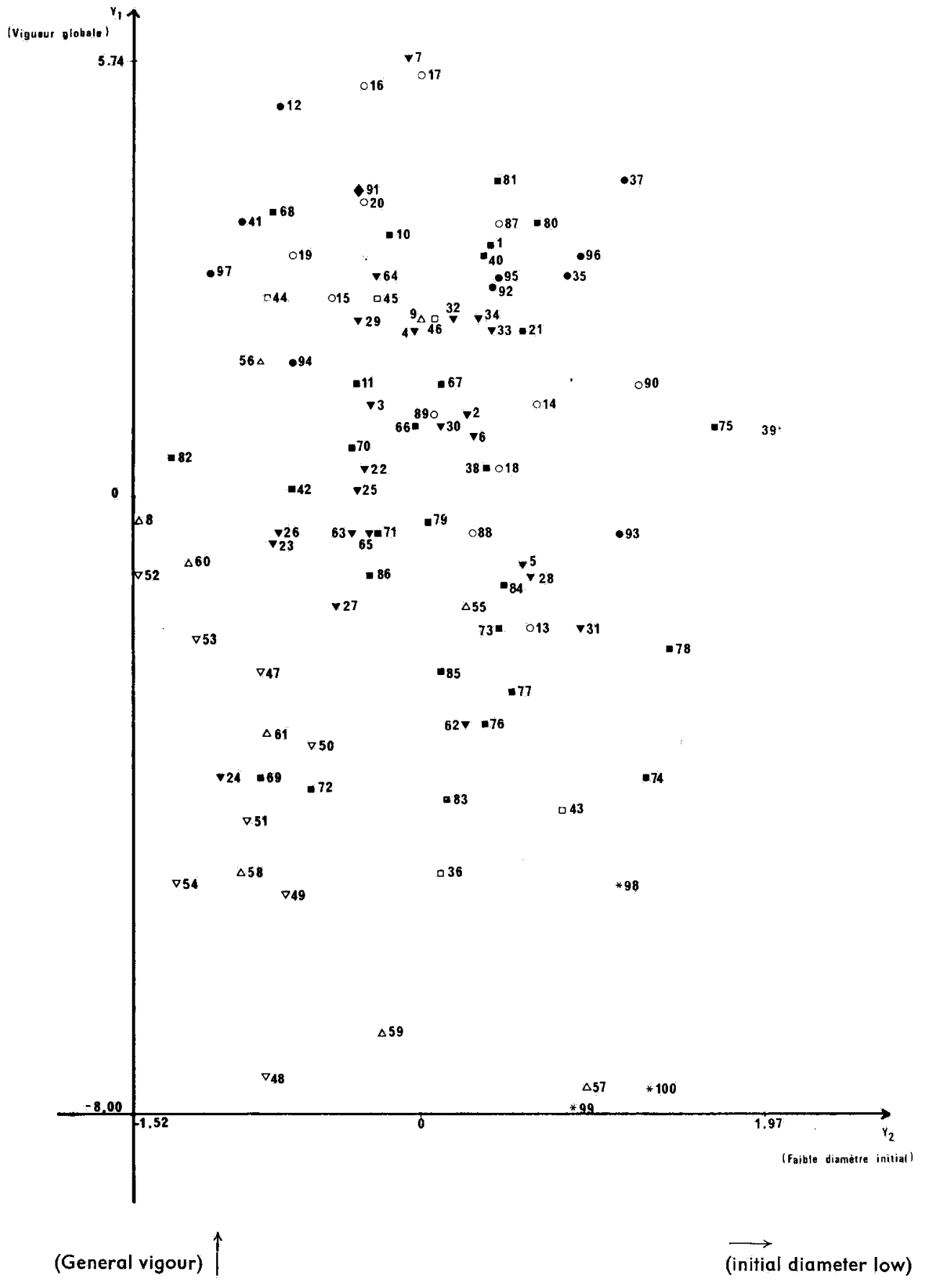


Le deuxième axe $\left(Y_{2}\right)$, ne prend plus en compte que 5 p. 100 de la variation totale, il est corrélé négativement avec le diamètre 1966 : il sépare les provenances qui, à « vigueur globale » équivalente (incluant déjà le diamètre 66), se différenciait par leur diamètre moyen initial (rappelons que les deux composantes sont indépendantes par construction) : sur la partie gauche du graphique se situent donc les provenances qui, pour un niveau de vigueur donné, ont un diamètre initial plus élevé. On trouve dans ce groupe les provenances alpines (Suisses surtout) qui, de par leur forme plus trapue s'opposent ainsi aux provenances nordiques de même vigueur globale peu élevée mais ayant une forme initiale plus élancée. On notera l'étalement des provenances autrichiennes selon la première composante surtout (forte variabilité de la vigueur globale), des provenances allemandes selon les deux composantes, des provenances des Beskides (Pologne et Moravie) enfin qui se distinguent par un haut niveau de vigueur globale avec une légère variation dans les diamètres initiaux.

\subsection{Comportement individuel.}

Dès 1971 (soit 5 ans après la plantation), les plants semblent sortis de leur éventuelle crise de transplantation puisque les corrélations entre hauteurs totales individuelles sont très élevées (0,81 entre hauteur 1971 et hauteur 1978). Par contre, les mesures individuelles en pépinière prédisent moins bien les performances individuelles en plantation : aussi bien entre hauteurs qu'entre circonférences, les corrélations pépinière-plantation ne sont que de 0,45. La crise due au repiquage, masquée au niveau provenance comme on l'a vu plus haut, reste néanmoins sensible au niveau individuel. La hauteur totale, 17 ans après plantation, est un très bon estimateur du volume individuel puisque la corrélation avec la circonférence à 1,30 $\mathrm{m}$ est très élevée $(0,87)$.

L'importance des conditions climatologiques très variables pendant la période étudiée d'une part, des effets de fertilité des blocs dont l'évolution a été mise en évidence plus haut d'autre part, contribuent sans doute à expliquer le manque de stabilité dans les croissances annuelles individuelles.

\begin{tabular}{|c|c|c|c|}
\hline 1972 et 71 & 1973 et 72 & 1974 ef 73 & 1975 ef 74 \\
\hline 0,56 & 0,48 & 0,52 & 0,48 \\
\hline $\begin{array}{c}1976 \text { ef } 75 \\
0,31\end{array}$ & $\begin{array}{c}1977 \text { ef } 76 \\
0,24\end{array}$ & $\begin{array}{c}1978 \text { et } 77 \\
0,0\end{array}$ & \\
\hline
\end{tabular}

On retrouve là encore une baisse très nette des corrélations entre deux années successives dès que l'année 1977 intervient. Indépendamment de cette année, les autres corrélations ne sont pas très élevées ( 0,5 en moyenne). Peut-être le niveau individuel inclut-il des interactions bloc-individu particulièrement importantes ici de par la grandeur de l'effet bloc lui-même. 


\section{2. - Les crifères de débourrement}

En 1973, 19 jours séparent la dernière notation de la première : lors du premier passage, la note moyenne est de 1 (bourgeon gonflé). Au second passage, elle est de 1,8 (apparition des aiguilles) et atteint 3,8 (stade pinceau) au dernier passage.

\subsection{Etude du milieu.}

L'effet bloc est ici encore très important, et de plus, au niveau des hauteurs totales, les blocs les plus vigoureux sont aussi les plus précoces. Ceffe liaison s'explique aisément par l'effet de lisière évoqué plus haut : si la lisière joue un rôle favorable sur la croissance, elle tamponne aussi les températures ef augmente donc la précocité des blocs voisins.

Par contre la liaison entre précocité d'un bloc et longueur de la pousse annuelle est beaucoup plus fluctuante, ceci étant dû essentiellement à l'inversion des classements de fertilité des blocs de 1974 à 1978 : jusqu'en 1974 la corrélation précocitélongueur de la pousse est positive $(0,6)$, les blocs de la partie basse, dans une zone plus froide et subissant une forfe concurrence sont tardifs et peu vigoureux alors que les blocs du haut, protégés par la lisière, sont vigoureux et précoces.

En 1975, cette corrélation s'annule et diminue progressivement pour atteindre 0,79 en 1978, la fertilité des blocs s'étant inversée alors que les zones basses sont toujours plus froides donc plus tardives.

\subsection{Efude des provenances.}

La première note a permis de déceler les provenances particulièrement précoces puisqu'en moyenne 12 p. 100 des plants seulement ont dépassé le stade 2 (apparition des aiguilles), ce pourcentage variant d'une provenance à l'autre entre 0 p. 100 et 44 p. 100 (provenance 3 - autrichienne).

Au dernier passage (deux semaines plus tard), 69 p. 100 des plants ont une note égale à 4 ou 5 (allongement maximum des aiguilles), mais pour certaines provenances ce pourcentage atteint 99 p. 100 alors que pour d'autres, particulièrement tardives, il n'est que de 25 p. 100 (provenance 60 - française - ou 99 - finlandaise).

Afin de prendre en compte l'ensemble des trois notations à la fois, on a voulu représenter globalement les provenances au moyen d'une analyse en composantes principales : les variables initiales sont les trois notes (au niveau moyennes de provenances) et les pourcentages définis plus haut.

La première composante (axe vertical $Y_{1}$ sur la fig. 2) absorbe 85 p. 100 de la variation totale, la deuxième composante en absorbe encore 10 p. 100.

La première composante, fortement corrélée avec toutes les variables initiales, est donc liée à la précocité générale des provenances : en haut du graphique on trouve les provenances autrichiennes particulièrement précoces, au milieu le groupe des provenances allemandes, tandis que les provenances du Nord-Est de la Pologne, quelques provenances alpines et des provenances des Beskides se distinguent par leur tardiveté.

La deuxième composante est corrélée négativement avec le premier pourcentage et positivement avec le dernier : elle peut être associée à la rapidité du débourrement. 
Pour un niveau de débourrement donné, on trouve à droite des provenances qui, relativement tardives au premier passage, ont débourré très vite entre le premier et le dernier passage (donc faible variabilité intraprovenance). A gauche au contraire, les provenances sont plus variables car, ayant commencé à débourrer plus tôt, elles se révèlent plus tardives lors du dernier passage.

Cette représentation permet de savoir comment s'exprime la tardiveté d'une provenance : ce peut être par un long étalement de la période de débourrement (provenances tchèques 15 et $18: 14$ p. 100 des plants ayant commencé à débourrer lors du premier passage, 49 p. 100 seulement sont complètement débourrés - note supérieure à 4 - lors du dernier) ou ce peut être encore par un début tardif de cette période (provenances alpines 57, 48 et 53 : premier pourcentage 0 p. 100, dernier pourcentage : 80 p. 100).

On retrouve ici les observations faites par Weisgerber ef al. 1976 : les provenances suisses, une partie des provenances françaises ef allemandes (provenances d'altitude en général) sont assez homogènes dans l'expression individuelle du débourrement (à droite sur la fig. 2). Par contre, le Nord-Est Polonais, la plupart des provenances des Beskides, plusieurs provenances françaises et allemandes se révèlent à la fois tardives et variables ce qui est très favorable à une amélioration de ce critère par sélection individuelle. La rapidité du débourrement pourrait être, à côté de la tardiveté, un nouveau critère de sélection, dans la mesure où elle raccourcit la période critique de sensibilité aux gelées tardives.

\section{3. - Liaisons vigueur-débourrement}

Dans l'échantillon des cent provenances représenté ici, aucune liaison significative entre les critères de débourrement et les critères de vigueur ne semble se dégager : une faible corrélation $(0,25)$ existe entre la précocité moyenne d'une provenance et la longueur de la pousse annuelle des années $1973,1974,1976$ ef 1978 . Avec la pousse de l'année 1975, la corrélation est nulle. Le comportement des provenances autrichiennes en 1977 déjà signalé plus haut, à savoir, une faible vigueur cette année-là alors que globalement les provenances sont précoces, explique sans doute la diminution brusque du coefficient de corrélation précocité-pousse en $1977(0,3)$. Là encore, une analyse en composantes principales permettra une représentation synthétique des provenances,

FIG. 2. - Critères de débourrement.

Représentation des provenances suivant les deux premières composantes principales.

Flushing traits. Distribution of provenances according to the two first principal components.

LÉGENDE : Roumanie
Tchécoslovaquie o
Autriche
Pologne
Allemagne
France $\Delta$
Suisse $\nabla$
Suède
Russie-Finlande $*$.


ÉPICÉA : VARIABILITÉ INTRASPÉCIFIQUE

121

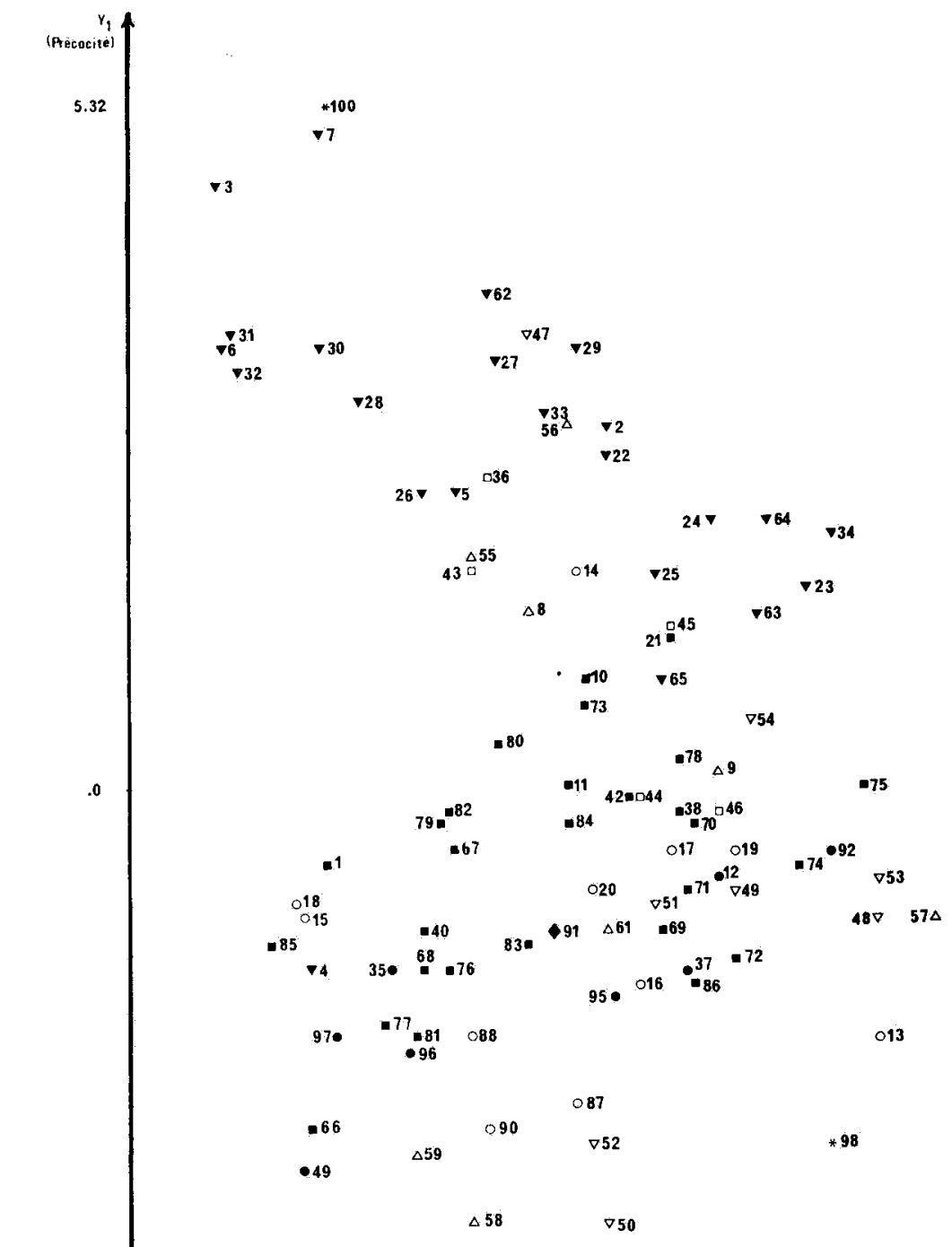

39

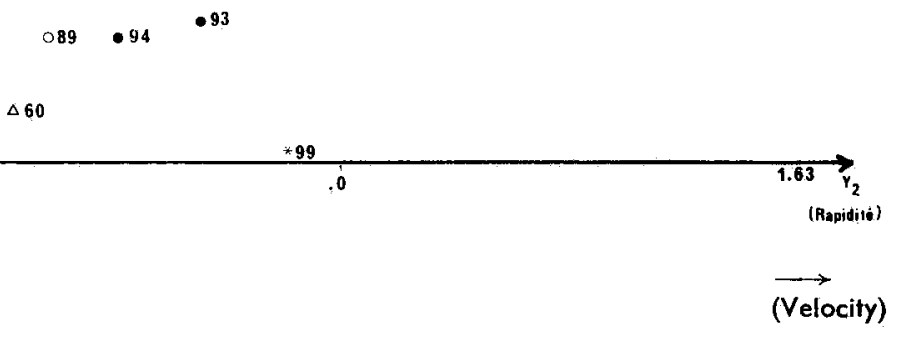

Annales des Sciences forestières. - 1980

9 
leur proximité sur le plan des deux premières composantes (fig. 3) traduisant ]eur ressemblance génotypique sur l'ensemble des critères de vigueur et de précocité : l'axe vertical, qui prend en compte 76 p. 100 de la variabilité totale est fortement corrélé $(0,9)$ avec toutes les mesures de hauteurs totales, de pousses annuelles et de circonférence.

Du haut en bas de la figure 3, les provenances se rangent donc par vigueur globale décroissante. Le deuxième axe qui absorbe encore 14 p. 100 de la variabilité, est fortement lié aux critères de précocité du débourrement $(0,9)$, mais révèle également une liaison négative avec la pousse $1977(0,4)$ : en effet, à droite sur le graphique se regroupent les provenances autrichiennes qui, quelle que soit leur vigueur globale, sont d'une part, précoces, et d'autre part, peu vigoureuses en 1977.

Dans la partie en haut à gauche de la figure 3 , les provenances à la fois tardives et vigoureuses, sont d'un grand intérêt pour les reboisements en France : il s'agit, de la provenance Roumaine (91), des provenances des Beskides, très vigoureuses et moyennement tardives (en Moravie : Vizovice, numéros 16 et 17 - en Pologne : Istebna-Wilsa, numéros 12, 35 et 37) ou des provenances du Nord et Nord-Est Polonais, moins vigoureuses mais plus tardives (Olstyn : 41 ou Bialowieza : 94) ainsi que des provenances tchèques (Cikhaj : 87, Sorcina Hrabusice : 90 ). Notons la grande variabilité de la provenance Kizovice (Moravie) qui, en plus des numéros 16 et 17, est également représentée par les numéros 14,15 et 18 , tous les trois beaucoup moins vigoureux : cette provenance est donc assez mal définie de par sa forte instabilité. Une provenance artificielle allemande (81: Ulzen) est particulièrement intéressante, elle se distingue de toutes les provenances allemandes par son comportement à la fois vigoureux et tardif. Bien que moins vigoureuse, la provenance Anzig (66) est également à signaler pour sa tardiveté. Le reste des provenances allemandes s'étale du haut en bas du graphique, toujours plus tardives que les autrichiennes de même vigueur, mais plus précoces que des provenances des Beskides. Parmi les provenances peu vigoureuses, leur précocité moyenne les situe au milieu des provenances alpines qui peuvent être, ou très fardives (60: Magland, Haute-Savoie) ou très précoces (47:Casis, Suisse) à des altitudes pourtant comparables. Trois provenances suédoises $(44,45,46)$ sont proches des meilleures provenances allemandes, les deux autres $(43,36)$ étant au contraire très peu vigoureuses et légèrement précoces.

FIG. 3. - Débourrement ef vigueur.

Représentation des provenances suivant les deux premières composantes principales.

Growth and Flushing traits

LÉGENDE : Roumanie
Tchécoslovaquie o
Autriche
Pologne
Allemagne
France $\Delta$
Suisse $\nabla$
Suède $\square$
Russie-Finlande *.




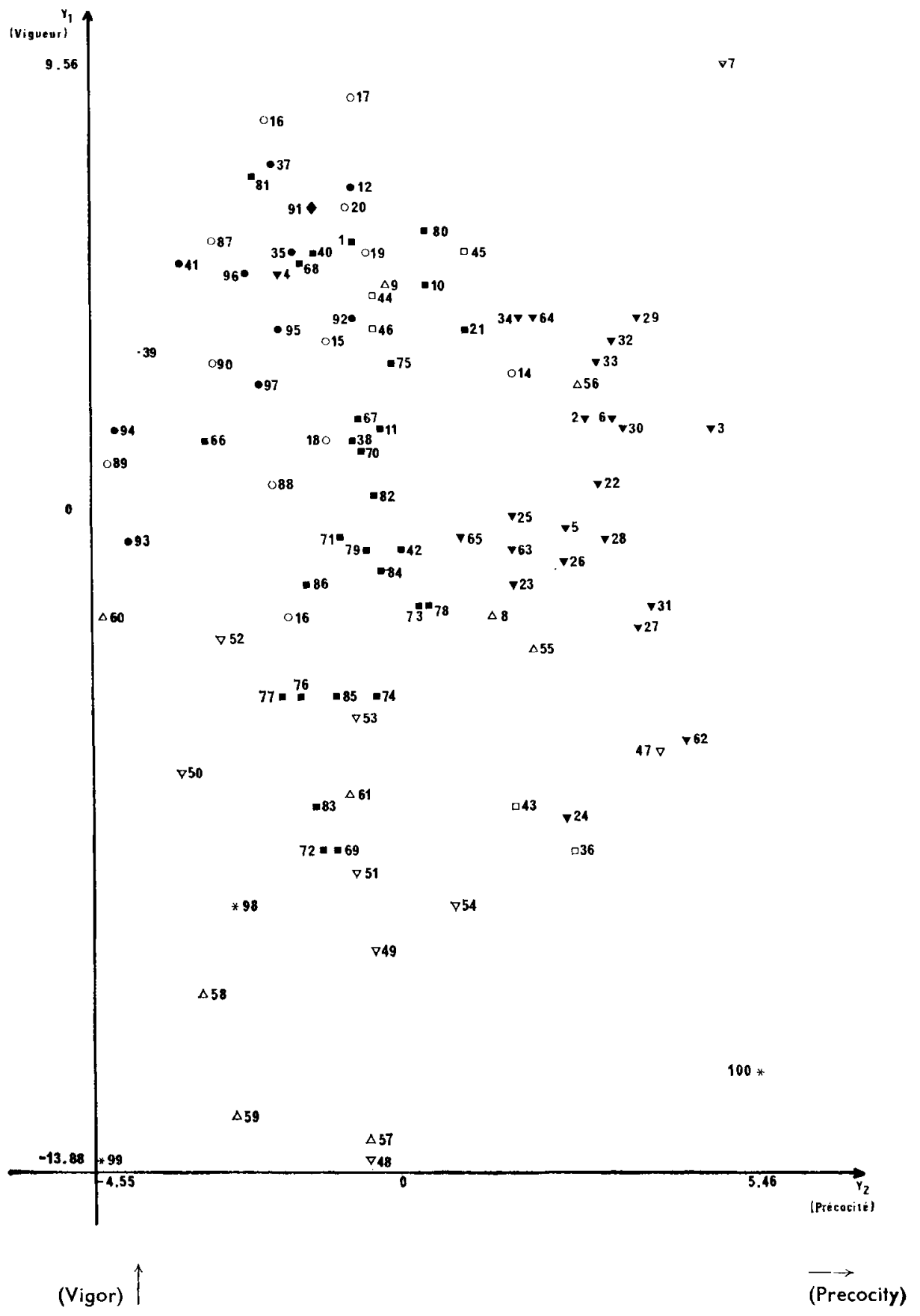


Au niveau des grandes régions géographiques, ces résultats confirment les classements généralement adoptés pour l'épicéa, que ce soit au niveau pépinière (Krutzsch, 1968) ou au niveau plantation (Gierfych, 1978 ; Krutzsch, 1974 ; Weisgerber ef al., 1977). Ils mettent l'accent sur certaines régions dont l'intérêt, pour le reboisement d'une part et pour une sélection individuelle d'autre part, est incontestable.

La réglementation établie dans le cadre de la C.E.E., rend particulièrement intéressant, l'étude du comportement en France des provenances allemandes.

\section{3. - Les régions de provenances allemandes}

\section{1. - La législation allemande}

D'après la loi allemande sur les plants et matériels de reproduction forestiers du 31.7.1972, le territoire de la République Fédérale Allemande est divisé en régions de provenances plus ou moins grandes, entièrement délimitées dans l'espace (voir fig. 4). Des semences récoltées à l'intérieur d'une région sont regroupées et commercialisées sous le numéro de cette région. L'application de cette loi rend impossible l'accès à des provenances individualisées, bien que, comme chez beaucoup d'espèces forestières, de nombreux peuplements d'épicéa génétiquement supérieurs jouissent dans la pratique d'un intérêt particulier. Pour tenir compte de ce fait, on a eu la possibilité de choisir des provenances ayant révélé de bonnes performances, de les récolter séparément ef de les commercialiser sous la désignation de la provenance après des contrôles d'homogénéité génétique du matériel. Ce type de peuplements sélectionnés est désigné sous le terme de «Sonderherkunft» ou « provenance spéciale 》. Si des tests appropriés établissent leur supériorité, ils pourraient être commercialisés au sein de la C.E.E. comme matériel certifié. L'introduction en France de matériel forestier allemand est actuellement limité au système des régions de provenances.

Le but de ce chapiirre est d'étudier, à partir des provenances allemandes représentées dans cette expérience (indiquées par des numéros sur la carte) et dans une expérience plus ancienne (indiquées par des lettres, Christophe, 1973), la stabilité des provenances par comparaison avec leur comportement en Allemagne d'une part, et l'homogénéité de régions de provenances introduites en France d'autre part.

\section{2. - Le classement des provenances et régions de provenance}

En s'appuyant uniquement sur des mesures de hauteurs totales obtenues dans plusieurs dispositifs à des âges différents, Schmidt-Vogt (1976 ef 1977) propose un classement global des régions de provenance, les performances de chaque provenance (dans chaque dispositif) étant exprimées en écart à la moyenne du dispositif ramenée à 100. La moyenne générale, sur tous les dispositifs, est également ramenée à 100 , ef c'est par rapport à cette moyenne que sont calculées les valeurs présentées dans le tableau 5. Les résultats des deux expériences françaises ont été reportés selon le même principe : dans ce qui suit, ces valeurs seront indiquées entre parenthèses après le nom des provenances.

En tête du classement, la région Westerhof domine nettement dans le classement allemand, elle est d'ailleurs reconnue comme le meilleur peuplement allemand (Weisgerber, 1977). Cependant les deux provenances représentées en France sont 


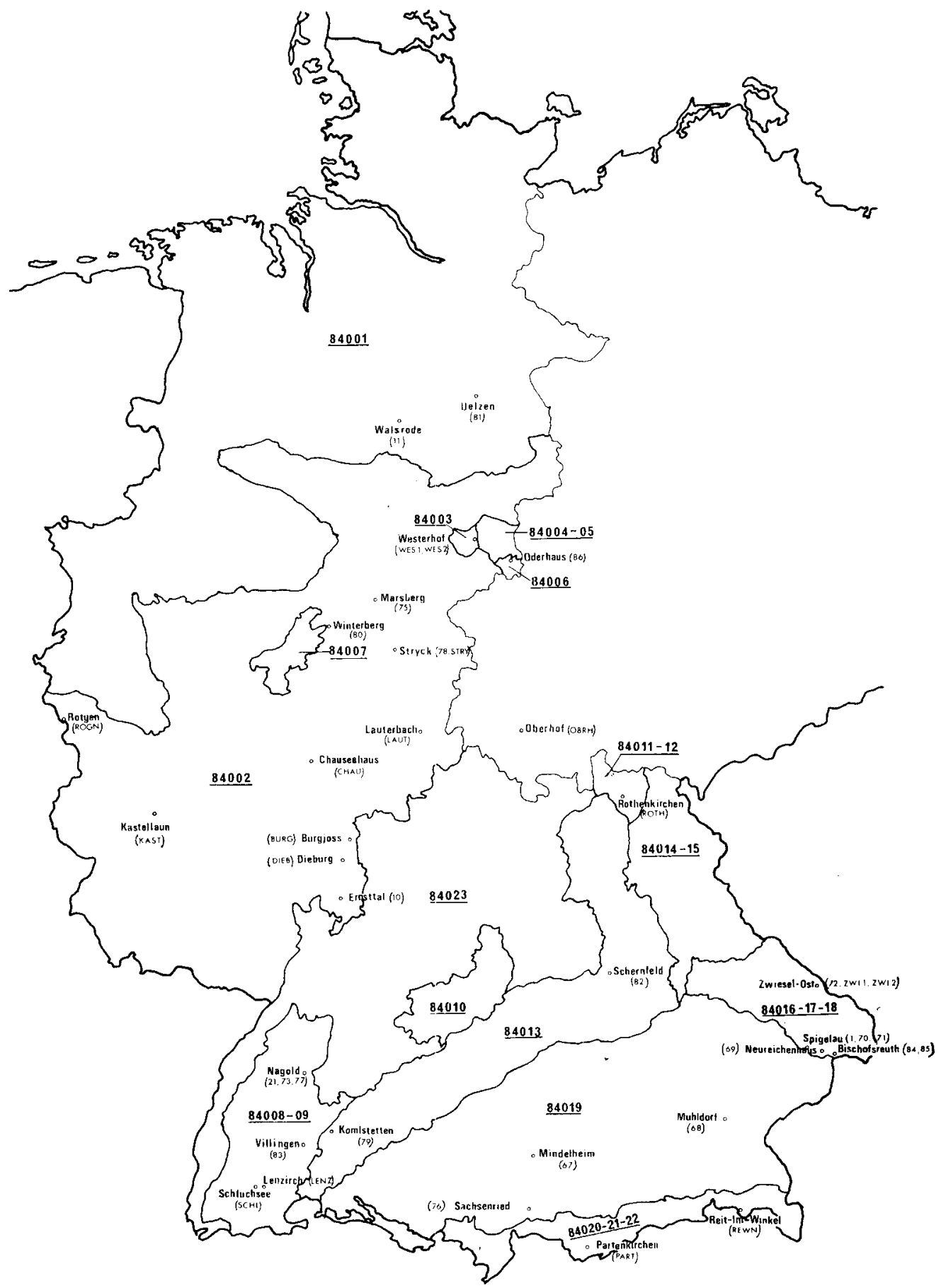

FIG. 4. - Carte des régions de provenances allemandes Map of german regions of provenances 
TABLEAU 5

Classement des régions sur la hauteur totale $100=$ moyennes générales des dispositifs Classification of german regions for fotal height $100=$ general mean

\begin{tabular}{|c|c|c|}
\hline \multirow[t]{2}{*}{$\begin{array}{l}\text { Numéro de la région } \\
\text { (numero of region) }\end{array}$} & $\begin{array}{l}\text { Résultats } \\
\text { français } \\
\text { (french results) }\end{array}$ & $\begin{array}{c}\text { Résultats publiés } \\
\text { par Schmidt-Vogt } \\
\text { (1976) } \\
\text { (results from } \\
\text { Schmidt-Vogt } \\
(1976) \text { ) }\end{array}$ \\
\hline & \multicolumn{2}{|c|}{$\begin{array}{l}\text { entre parenthèses : } \\
\text { nombre de provenances } \\
\text { (between bracketts: } \\
\text { number of provenances) }\end{array}$} \\
\hline 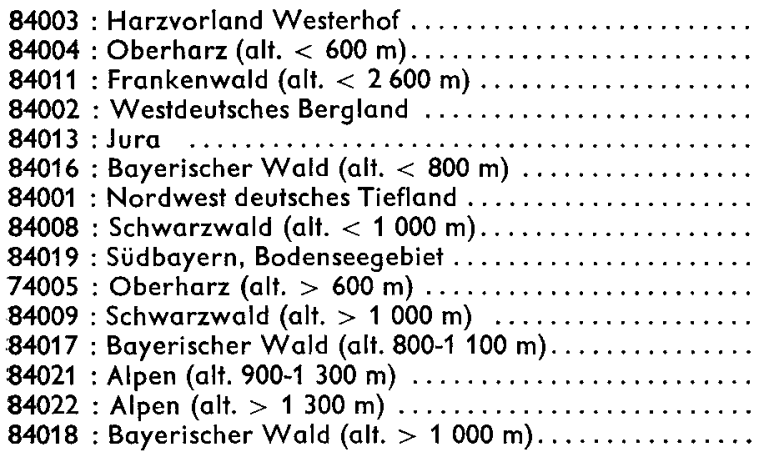 & $\begin{array}{l}110(2) \\
106(2) \\
105(1) \\
106(11) \\
99(2) \\
111(1) \\
109(2) \\
94(6) \\
102(4) \\
95(3) \\
81(1) \\
100(2) \\
100(1) \\
102(1) \\
91(6)\end{array}$ & $\begin{array}{l}123(3) \\
112(1) \\
111(3) \\
108(22) \\
107(24) \\
105(16) \\
103(2) \\
103(8) \\
99(55) \\
96(2) \\
91(15) \\
94(5) \\
92(2) \\
80(2) \\
78(15)\end{array}$ \\
\hline
\end{tabular}

peu comparables puisque l'une atteint la valeur la plus grande (120) alors que l'autre n'est qu'à 100 (moyenne générale).

Viennent ensuite dans le classement allemand, les régions du Harz (84004) et de Frankenwald (84011) de basse altitude, également vigoureuses en France et dans les dispositifs de Weisgerber. La provenance Rothenkirchen (84011) est intéressante à signaler de par sa stabilité de comportement (105 en France, 106 et 116 en Allemagne). Cependant en France, une région a surpassé les précédentes : il s'agit du bas pays au Nord (84001 : 109), dont une provenance Ulzen (114) également remarquée par Weisgerber, a déjà été signalée plus haut. Cette région représentée par deux autres provenances moins vigoureuses chez Schmidt-Vogt, est moins bien classée en Allemagne (103).

La région 84002, très étendue sur le terrain, a un comportement moyen aussi vigoureux en Allemagne qu'en France (108 et 106). Il faut écarter la provenance Stryck, qui est très bien classée en Allemagne (110) et signalée comme très vigoureuse par Weisgerber : elle est par ailleurs une « Sonderherkunft ». Pourtant en France, représentée dans les deux expériences, elle a une croissance inférieure à la moyenne : 96 et 94 , son introduction est donc à déconseiller. Dans cette région, quelques provenances se révèlent en France très vigoureuses: Rötgen (107), Chausseehaus (116) ef Dieburg (117), ces deux dernières étant remarquées également par Weisgerber. La «Sonderherkunft » Burgjoss, atteint 102 en France et 115 en Allemagne. 
La région 84016 (Forêt bavaroise, basse altitude) a un bon comportement moyen (111 et 107) : elle est représentée en France par une provenance Spiegelau assez stable puisqu'elle atteint 111 en France ef 109 chez Weisgerber.

La région 84013 s'est révélée moins performante en France qu'en Allemagne, ceci étant dô à la différence d'échantillonnage : la partie Nord de cette région appartient à l'aire naturelle de l'épicéa et est très vigoureuse en général (110 à 120). La provenance Schernfeld, représentée ici, a justement la plus faible valeur (110) chez Schmidt-Vogt. La seconde provenance est située dans la partie Sud de cette région, reconnue comme moins vigoureuse.

La région 84008 (Forêt Noire, altitude inférieure à $1000 \mathrm{~m}$ ), ne se révèle pas a ussi vigoureuse en France (94) qu'en Allemagne (103). Elle englobe ici trois provenances Nagold assez inégales (107, 95 et 93), et des provenances Lenzkirch et Höhenfichten des Schwarzwaldes (89 ef 93), classées comme «Sonderherkünfte » à une altitude supérieure à $1000 \mathrm{~m}$ (région 84009).

Au contraire, deux régions d'altitude, 84020 et 84022 (Alpen), représentées par Reit-im-Winkel (900-1000 m) ef Partenkirchen (1600-1 $700 \mathrm{~m}$ ), ont une performance moyenne en France (100 et 102) alors que ces régions sont généralement considérées comme très peu vigoureuses en Allemagne.

La région 84019 a un comportement moyen dans les deux pays (102 ef 99) : 18 stations de la provenance Sachsenried, représentées en Allemagne, sont très variables et certaines peu vigoureuses $(81,79$ ef 68$)$.' Cette provenance atteint 93 en France. Les autres sont plus vigoureuses : Mühldorf (110), Mindelheim (103 à côté de 112 en Allemagne) et Anzig (103).

Signalons la bonne stabilité de la région 84005, représentée dans les deux pays par deux provenances Oderhaus, classée comme «Sonderherkunft» Autochtone Harzfichte : elles réalisent 95 ef 98 en Allemagne et 91 ef 97 en France.

La région 84017 est représentée par deux provenances en France : Zwiesel (98) et Spiegelau (102). La provenance Zwiesel, représentée quatre fois en Allemagne n'atteint que 88 en moyenne ( 83 à 94 ).

Deux régions d'altitude se situent en fin de classement, mais leur comportement diffère d'une expérience à l'autre : la région 84018 (Forêt bavaroise, altitude supérieure à $1000 \mathrm{~m}$ ) est considérée par Schmidt-Vogt comme beaucoup moins vigoureuse que la région 84009 (Forêt Noire, même altitude) : 78 pour la première, 91 pour la seconde. En France, le comportement moyen des provenances de la Forêt bavaroise se rapproche des observations de Weisgerber : Spiegelau (99 ef 109), Bischofsreuth (97 et 93 d'un côté, 92 et 105 de l'autre), enfin Neureichenhaus (86 et 87). Par contre, Spiegelau ne fait plus que 69 chez Schmidt-Vogt, il en va de même pour Zwiesel-Ost $\checkmark 1,2$ (86 d'un côté, 69 de l'autre).

La seule provenance de la Fôrêt Noire représentée en France, Schluchsee a une croissance aussi faible dans les deux dispositifs (81 et 83). Mais en Allemagne, d'autres provenances plus vigoureuses remontent la moyenne de la région 84009 .

Globalement, malgré la diversité des dispositifs installés aussi bien en France qu'en Allemagne, les régions du Harz (84003 et 84004) et de Frankenwald (84011), la grande région centrale 84002 et la Forêt bavaroise de basse altitude (84016) sont des régions considérées comme vigoureuses. Les régions de la Forêt Noire (84008 et 
84009) poussent moins bien en France qu'en Allemagne, alors que les trois régions d'altitude $(84020,84022$ et 84018$)$ donnent de meilleures performances en France.

Pour les critères de débourrement, Weisgerber signale la tardiveté de quelques provenances de la Forêt bavaroise, Spiegelau ef Zwiesel-Ost, tardiveté déjà constatée pour Zwiesel (Chrisłophe, 1973) mais que l'on ne retrouve pas ici : les provenances 72 (Zwiesel) et 71 (Spiegelau) onf effectivement débourré tard (3 p. 100 seulement de plants débourrés au premier passage, alors qu'en moyenne sur les 100 provenances il y en avait 12 p. 100) mais très vite puisqu'au troisième passage 70 p. 100 des plants déjà avaient atteint le stade 4.

Dans le Nord, Winterberg, Stryck ef Ulzen sont également classées comme tardives en Allemagne mais seule Ulzen reste tardive en France.

Signalons la région 84019 (Südbayern) qui dans cette étude est représentée par 4 provenances, toutes tardives. Mühldorf, à la fois vigoureuse et tardive, forme avec Spiegelau (n०1) et Ulzen un groupe de provenances intéressantes pour le reboisement de la région lorraine.

\section{3. - L'homogénéifé des régions de provenances}

Nous reprenons rapidement les résultats établis sur deux régions, par Christophe, 1973 : la région Westerhof (84003) était représentée par deux provenances, dont l'une était très vigoureuse ef tardive et l'autre de vigueur moyenne et très précoce.

La région 84002 était représentée par six provenances, dont trois très vigoureuses ef trois de vigueur moyenne. Parmi les trois premières, Rötgen et Dieburg étaient tardives et Chausseehaus précoce. Parmi les trois dernières, on signale la précocité de Stryck.

Ces résultats posaient déjà le problème de la notion de régions de provenances telle qu'elle avait été définie par le décret allemand du 31.7.1972. Il est en effet difficile d'admettre, pour le reboisement en France, un mélange de provenances telles que Rötgen et Stryck.

La figure 5 présente les vingt-huit provenances allemandes selon leur hauteur totale 1978 et leur précocité de débourrement (pourcentage de plants ayant atteint le stade « pinceau » au dernier passage) : les provenances sont regroupées selon leur appartenance d̀ une région de provenances. Cette représentation pose clairement le problème de l'homogénéité des régions :

- la région 84002, déjà signalée plus haut, comporte quatre provenances, dont trois sont vigoureuses et la dernière (Stryck) a une vigueur inférieure à la moyenne.

- la région 84019, représentée ici par quatre provenances toutes assez tardives est hétérogène pour la vigueur : la provenance Mühldorf présente une supériorité relative de 18 p. 100 par rapport à la provenance Sachsenvied.

- la région 84018 regroupe une provenance très tardive et peu vigoureuse (Bischofsreuth 85) et quatre provenances de précocité moyenne, dont deux également de vigueur moyenne (Bischofsreuth 84 et Spiegelau 81) et deux très peu vigoureuses (Neureichenhaus 69 et Zwiesel Ost 72).

- la région 84008 enfin, la plus hétérogène dans cette expérience, inclut trois provenances Nagold dont l'une (21) est vigoureuse et extrêmement précoce (83 p. 100 de plants débourrés) et les deux autres sont de vigueur inférieure à la moyenne (14 p. 100 plus faibles que la première), mais de précocité différente : Nagold 73 a 


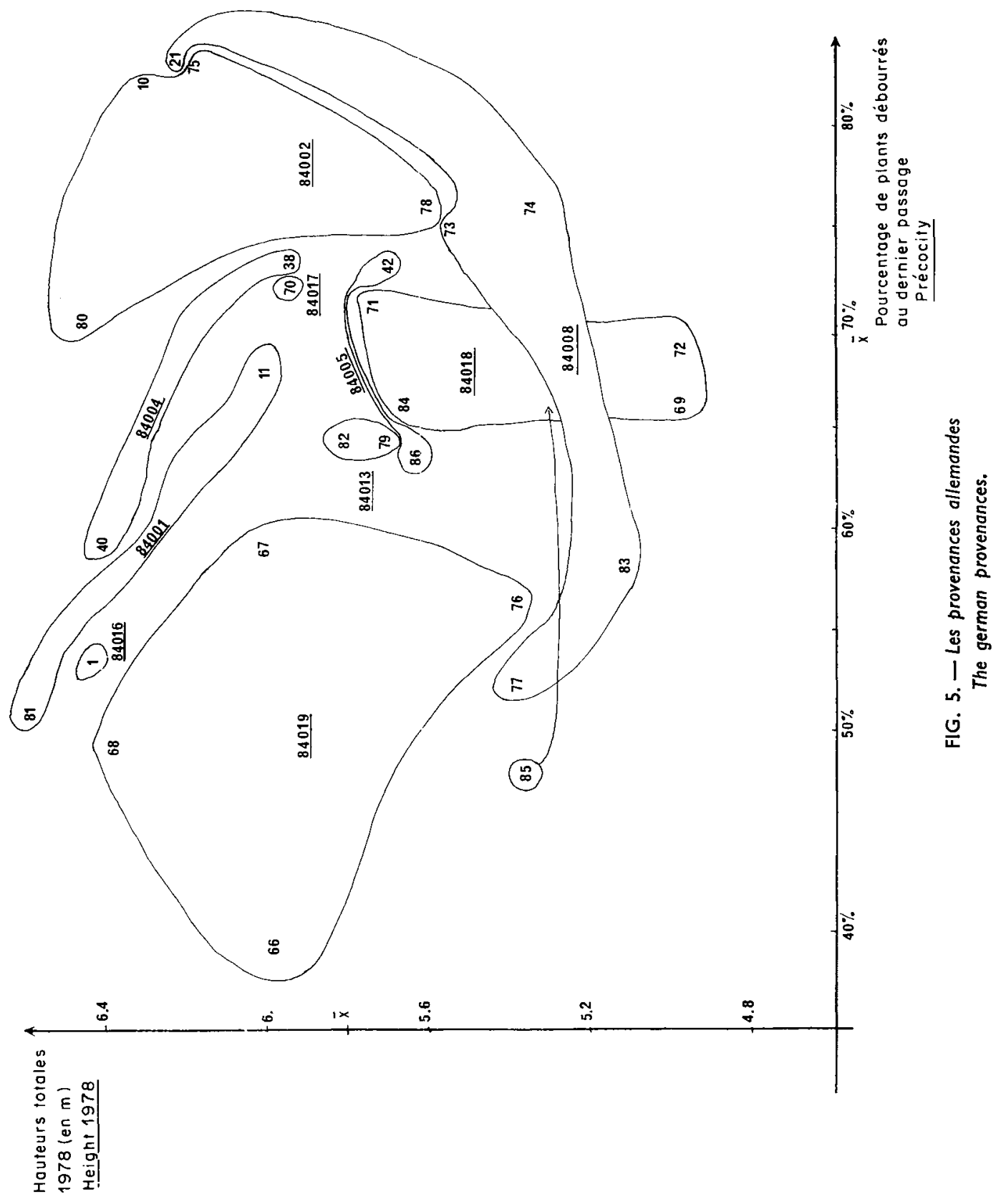


déjà 75 p. 100 de plants débourrés, Nagold 77 n'en a que 51 p. 100 . A cette région appartiennent aussi les provenances Höhenfichten des Schwarzwaldes (74), proche de Nagold 73 et Villingen (83) proche de Nagold 77.

Il est donc évident, d'après ces résultats, que la notion actuelle de « régions de provenances allemandes » doit être redéfinie : l'utilisation de cette notion implique qu'il y ait une certaine homogénéité du matériel issu d'une même région (CTGREF, 1976), ce qui n'est pas le cas actuellement.

\section{4. - Discussion et conclusion}

A partir des résultats exposés dans les chapitres précédents, nous retracerons brièvement ici les éléments importants de notre étude concernant la variabilité géographique de l'épicéa :

- au niveau de grandes régions géographiques, le classement de ces régions est relativement stable d'un pays à l'autre, avec une supériorité en vigueur des régions des Beskides (Moravie et Pologne) jointe à une bonne tardiveté du débourrement, et une très bonne tardiveté du Nord-Est Polonais jointe à un comportement vigoureux ;

- au niveau plus fin des provenances, des interactions importantes sont parfois à signaler : ainsi la provenance artificielle Stryck, bien classée en Allemagne, esł à bannir en France pour son manque de vigueur, ainsi les provenances de la forêt bavaroise d'altitude, Spiegelau ef Zwiesel-Ost, considérées comme très médiocres par Schmidt-Vogt alors que Weisgerber et nos résultats les considèrent comme moyennes ;

- au niveau de la provenance même, l'hétérogénéité de certains peuplements pose des problèmes importants pour leur utilisation : ainsi Schmidt-Vogt (1977) montre que la provenance Sachsenried est constituée par une mosaïque de populations allant de peu vigoureuses à très vigoureuses, et de précoces à tardives; ainsi la provenance Westerhof, reconnue partout comme très vigoureuse, dont un des peuplements représenté ici s'est révélé peu vigoureux; ainsi enfin la provenance Nagold (84008) dont on a signalé plus haut la forte hétérogénéité ;

- au niveau enfin de la région de provenances, les forestiers allemands ont euxmêmes déjà posé le problème de l'hétérogénéité des régions allemandes : ils l'ont résolu à leur niveau en bannissant l'utilisation de peuplements artificiels et en publiant des « Recommandations pour l'utilisation de provenances adaptées », d'après le comportement des provenances testé en plantations comparatives.

De plus, Schmidt-Vogt (1976), critique le mélange dans une même région, de peuplements autochtones ef de peuplements artificiels : c'est le cas en effet des régions 84008,84019 ef 84013 . II recommande de délimiter au Nord de la région 84013 une région autochtone et de regrouper les peuplements artificiels du Sud de la région 84013 et du Sud de la région 84019 , s'étendant alors jusqu'à la limite de l'aire naturelle dans le «Alpenvorland ». La partie hors de l'aire naturelle de la région 84008/09 (au Nord et à l'Ouest de la Forêt Noire) devrait également être isolée.

Remarquons que nos résultats confirment ces observations : Nagold, très hétérogène, est située dans cette partie Nord de la région 84008 , donc artificielle.

L'utilisation en France de ces Régions reste problématique, car les régions de l'aire naturelle de l'épicéa allemand se révèlent peu intéressantes (à part la 84011, représentée ici par une seule provenance, Rothenkirchen) : même la région Westerhof 
est remise en question. Certaines provenances, malheureusemenf d'origine inconnue, se sont par contre révélées excellentes, et par leur vigueur, et par leur tardiveté : il s'agit par exemple de Rötgen, Dieburg (84002) ou encore de Ulzen (84001), déjà recommandée par Weisgerber, et qui devraient pouvoir être commercialisées sans être mélangées avec des provenances telles que Walsrode (84001), précoce ou Stryck (84002), peu vigoureuse et précoce.

Ceci condamnerait dans le cas présent la notion allemande de région de provenances : il vaudrait mieux, malgré les difficultés pratiques, pouvoir commercialiser des peuplements sélectionnés, leur supériorité ayant été mise en évidence dans des plantations comparatives répétées dans plusieurs stations. Il s'agira alors de « matériel contrôlé » qui, nous l'espérons, prendra le relais des matériels sélectionnés.

Reçu pour publication en août 1979.

\section{Summary}

The intraspecific variation of Spruce (Picea abies) in its natural range was analysed from two experimental designs in France in comparison with similar designs in Germany.

At regions level the superiority of Beskides (Poland, CSSR), Romania and nord-eastern Poland was obvious in France as in Germany for growth and flushing traits.

At provenances level some provenance $x$ design interactions were found for growth traits.

A special attention was paid to german provenances and regions of provenances because of their commercial interest for the reforestation in France : it was clear that some provenances and the majority of regions are heterogeneous. The concept of region has been already criticized by the german forest breeders and should be revised : because of the too large area of these regions and the mixture of natural and artificial populations within the same region, it is impossible to guarantee the homogeneity of the material from a given provenances region.

\section{Références bibliographiques}

ANON. Empfehlungen geeigneter Herkünfte für den Anbau in Niedersachsen. Merkblatt, Niedersächsische Forstliche Versuchsantalt, Escherode (1974).

CHRISTOPHE, C. 1973. - Etude des provenances et régions de provenances allemandes. Centre National de Recherches Forestières. Station d'Amélioration des arbres forestiers, Doc. interne 73/6, $11 \mathrm{p}$.

C.T.G.R.E.F., 1976. Semences forestières. Les régions de provenances d'épicéa commun ; note technique $n^{\circ} 30$.

KRUTZSCH P., 1968. Pflanzenschulergebnisse eines inventierenden Fichtenherkunftsversuches (Picea abies und Picea obovata). ForstgenetischesInstitut Kgl. Forstl. Hochschule in StockholmSchweden, 47 p. + annexes.

KRUTZSCH P., 1974. The IUFRO 1964/68 provenances test with Norway Spruce (Picea abies (L.)). Silvae genetica $23,58-62$.

GIERTYCH M., 1978. Adaptability of polish spruce (Picea abies (L.) M. Karst) races in the light of the IUFRO 1964/68 international provenance experiment. En polonais : Arborełum KornickieRocznik XXIII.

SCHMIDT-VOGT, 1976. Fichtenherkünfte (Picea abies (L.) Karst) der Bundesrepublik Deutschland. Erste Auswertung der Versuchsreihe 1959/66. Allg. Forst U. J. Ztg., 147, 149-163.

SCHMIDT-VOGT M., 1977. Die Fichte. Band I : Taxonomie-Verbreitung ; Morphologie-OkologieWaldgesellschaften, Hamburg, Berlin : Parey, $647 \mathrm{p}$.

SEARLE S. R., 1971. Linear Models, John Wiley, New York, 532 p.

WEISGERBER M., DIETZE W., KLEINSCHMIT J., RACZ J., DIETERICH H. und DIMPFLMEIER R., 1976. Ergebnisse des internationalen Fichten-Provenienzversuches 1962. Teil I. Phänologische Beobachtungen und Höhenwachstum bis zur ersten Freiland aufnahme. Allg. Forst U. J. Złg., 147 (12), 227-235.

- 1977. Ergebnisse des internationalen Fichten Provenienzversuches 1962. Teil II : Weiter Entwicklung bis zum Alter 13. Allg. Forst U. J. Ztg., 148 (12), 217-227. 


\section{ANNEXE}

Liste ef localisation des cent provenances

Numéro
International
en séquence Pays d'origine $\quad$ Provenance

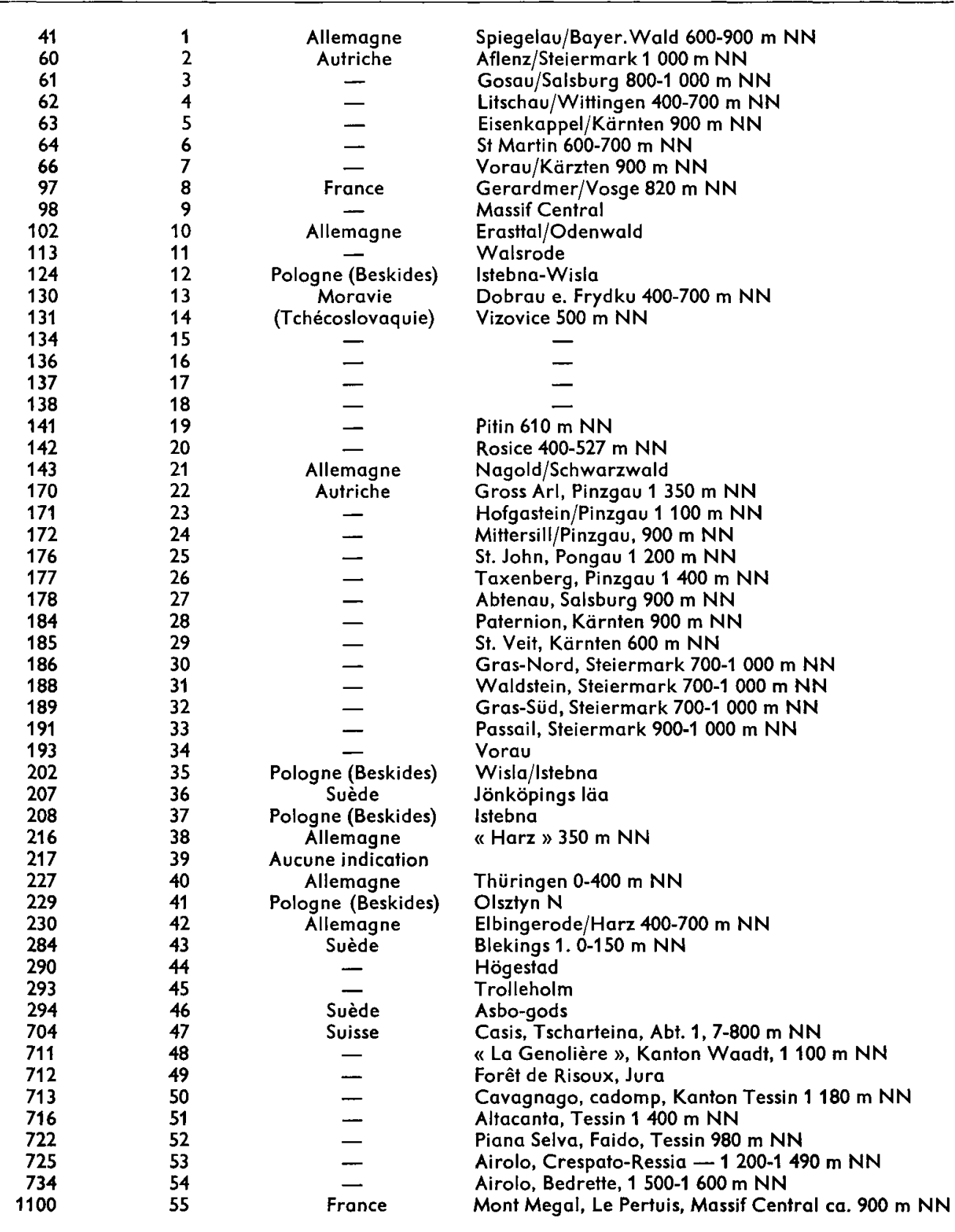


ANNEXE (suite)

\begin{tabular}{|c|c|c|c|}
\hline $\begin{array}{c}\text { Numéro } \\
\text { international }\end{array}$ & $\begin{array}{c}\text { Numéro } \\
\text { en séquence }\end{array}$ & Pays d'origine & Provenance \\
\hline
\end{tabular}

\begin{tabular}{|c|c|c|c|}
\hline 1101 & 56 & - & Le Pertuis, Monts du Velay 1100 m NN \\
\hline 1111 & 57 & - & Peisey-Nancroix, Grd. Bois $1400 \mathrm{~m}$ NN \\
\hline 1112 & 58 & - & Autrans, Isère, parcelle 22, 1250 m NN \\
\hline 1114 & 59 & - & Passy, Hte Savoie, parcelle $30,1200 \mathrm{~m} \mathrm{NN}$ \\
\hline 1115 & 60 & - & Magland, Hie Savoie, $700 \mathrm{~m} \mathrm{NN}$ \\
\hline 1116 & 61 & - & Lantosque, Alpes Maritimes, $1500 \mathrm{~m}$ NN \\
\hline 2113 & 62 & Autriche & Oberes Gailtal, Krnt. \\
\hline 2120 & 63 & - & Liembergwald, Zell a. S. \\
\hline 2122 & 64 & - & Piberegg, Stmk 750-850 m NN \\
\hline 2121 & 65 & - & Neukirchen, Zell a. S. \\
\hline 3203 & 66 & Allemagne & F.A. Anzig, $300-700 \mathrm{~m}$ \\
\hline 3212 & 67 & - & F.A. Mindelheim, $300-700 \mathrm{~m}$ \\
\hline 3213 & 68 & - & F.A. Mühldorf, 300-700 m \\
\hline 3326 & 69 & - & F.A. Neureichenhaus, über $1100 \mathrm{~m}$ \\
\hline 3328 & 70 & - & F.A. Spiegelau, unter $1100 \mathrm{~m}$ \\
\hline 3329 & 71 & - & F.A. Spiegelau, über $1100 \mathrm{~m}$ \\
\hline 3334 & 72 & - & $\begin{array}{l}\text { F.A. Zwiesel-Ost, Plattenf.-Einze l-st. Abt V/1 u. } 2 \text { über } \\
1100 \mathrm{~m}\end{array}$ \\
\hline 3414 & 73 & - & VIII/13 und $700 \mathrm{~m}$ (Nagold) \\
\hline 3416 & 74 & - & VIII 6 u. $1000 \mathrm{~m}$ Höhenfichten des Schwarzwaldes \\
\hline 3417 & 75 & - & VIII/2 Westdeutsches Bergl. Marsberg \\
\hline 3418 & 76 & - & VIII/14 Sachsenried \\
\hline 3419 & 77 & - & VIII/6 300-700 (Nagold) \\
\hline 3422 & 78 & - & VIII/2 Westdeutsches Bergland und $600 \mathrm{~m}$ Stryck \\
\hline 3423 & 79 & - & VIII/9 Jura FA Kohlstetten üb. 700 \\
\hline 3424 & 80 & - & VIII $/ 2$ ü. $600 \mathrm{~m}$ Winterberg \\
\hline 3426 & 81 & - & Saat Nr. $1736 \mathrm{VIII} / 1$ VoBmoor FA Uelzen \\
\hline 3428 & 82 & - & VIII/9 Jura FA Schernfeld \\
\hline 3429 & 83 & - & $\begin{array}{l}\text { Saat Nr. } 1440 \text { VIII/6 700-1 } 000 \mathrm{~m}, \text { VoBmoor FA Vil- } \\
\text { lingen }\end{array}$ \\
\hline 3435 & 84 & - & $\begin{array}{l}\text { VIII/11 Saat Nr. } 1794 \text { ü. } 1100 \mathrm{~m} \text { VoBmoor FA Bischofs- } \\
\text { reuth }\end{array}$ \\
\hline 3437 & 85 & Allemagne & Bischofsreuth \\
\hline 3438 & 86 & - & VIII/4 ü. $600 \mathrm{~m}$ Oderhaus \\
\hline 4011 & 87 & Moravie & Cikhaj, a. d. Svratka \\
\hline 4111 & 88 & Tchécoslovaquie & \\
\hline 4143 & 89 & - & Kysihybel. Lehrreivier (Einzelstamm) \\
\hline 4159 & 90 & - & Svrcina Hrabusice \\
\hline 5425 & 91 & Roumanie & $\begin{array}{l}\text { Cimpeni, "Nedei ", Valea Bistrei, Abt. } 51 \mathrm{~b} \text { u. c. } \\
1300-1500 \mathrm{~m}\end{array}$ \\
\hline 6106 & 92 & Pologne (Beskides) & Koszalin (Nord) \\
\hline 6224 & 93 & - & Czerwony Dwor (NE) \\
\hline 6236 & 94 & - & Bialowieza, 403, $150 \mathrm{~m}$ (NE) \\
\hline 6254 & 95 & - & Mikaszowka, $120 \mathrm{~m}(\mathrm{~N})$ \\
\hline 6255 & 96 & - & «Puszcza Augustowka » \\
\hline 6410 & 97 & - & Sierpe, $110 \mathrm{~m}$ (Centre) \\
\hline 7401 & 98 & U.R.S.S. & Gzatsk Revier, Smolensk \\
\hline 7411 & 99 & - & $\begin{array}{l}\text { Dedenonskoe Revier, } \\
\text { Dimitrovskogo FV }\end{array}$ \\
\hline 8707 & 100 & Finlande & $\begin{array}{l}\text { Janakkala, Keskivari } \\
\text { Uhkoila, Best. } 11\end{array}$ \\
\hline
\end{tabular}

